\title{
La cal libre de los cementos y la expansión en autoclave
}

\section{J. CALleJA* y C. del OLMO **}

\section{R E S U M E N}

Como continuación de un trabajo anterior, se exponen en éste criterios y puntos de vista relativos a la interpretación y valoración de los resultados del ensayo de expansión en autoclave de los cementos, en relación con las varias causas que pueden dar lugar a dicha expansión.

En particular, se considera el caso de la producida por la cal libre, a base de una numerosa serie de resultados experimentales, y se da una explicación más de la falta de correlación entre contenidos de cal libre y expansiones en autoclave.

Se sientan las bases para abordar en trabajos sucesivos el estudio de la expansión debida a la magnesia y a los aluminatos cálcicos cristalizados.

\section{INTRODUCCION}

Los principales componentes expansivos de los cementos, que normalmente pueden encontrarse presentes en una $u$ otra proporción en la composición de los mismos, son la cal libre, la magnesia libre (ésta última cristalizada en forma de periclasa) y los aluminatos cristalinos.

La cuantía de estos componentes depende de multitud de factores relativos a las materias primas, a la dosificación del crudo, a los procesos de cocción y enfriamiento del clínker, y al ensilado y aireación del cemento.

Tanto la cal como la magnesia libres del cemento son óxidos calcinados a muy alta temperatura -la de clinkerización: unos $1.450^{\circ} \mathrm{C}$-, por lo cual son muy lentos en su proceso de hidratación expansiva a la temperatura ordinaria. También lo son, en otra medida, los aluminatos cristalinos. Esta lentitud es la causa de que los procesos de la hidratación de ambos óxidos y de los aluminatos cristalizados no manifiesten sus efectos expansivos de forma inmediata, sino diferida, con lo cual la expansión produce tensiones en el mortero y en el hormigón de cemento, ya endurecido, que pueden crear serios problemas.

\footnotetext{
* Profesor de Investigación. Jefe del Departamento de Química del Instituto "Eduardo Torroja" de la Construcción y del Cemento.

** Colaborador Científico. De la Sección de Análisis y Ensayos del citado Departamento.
} 
La expansión de los cementos tiene, pues, un carácter potencial y se manifiesta o actualiza a plazo, en general, no corto. Por este motivo los ensayos para detectarla, o bien son lentos y se aceptan como tales, o bien han de ser acelerados.

El ensayo más rápido, y por lo tanto el más drástico en cuanto a las condiciones de su ejecución, es el ensayo norteamericano del autoclave, tal como figura en las normas ASTM (1).

En cuanto a las particularidades de este ensayo, a su comparación con otros menos severos y, sobre todo, a las limitaciones lógicas y previsibles de su campo de aplicación y de la validez de los resultados obtenidos mediante él, se ha informado con detalle y de forma crítica en otro lugar (2).

El ensayo de expansión en autoclave se basa en la medida de la longitud de una probeta, antes y después del mismo, y en la expresión de la diferencia entre ambos valores de la longitud como tanto por ciento de la longitud primitiva, antes de la expansión. Es, pues, un ensayo de carácter cuantitativo y por ello permite, "en principio", establecer comparaciones y relaciones, e incluso fijar limitaciones numéricas a la expansión máxima admisible de los cementos. Pero sólo en principio, en algunos casos.

\section{LIMITACIONES QUE AFECTAN A LA UTILIZACION DE LOS RESULTADOS DE EXPANSION DE LOS CEMENTOS EN AUTOCLAVE}

Un aspecto muy importante, y no suficientemente tenido en cuenta, de los resultados de la expansión en autoclave es la frecuente falta de correlación entre ellos y el comportamiento real de los cementos en la páctica. Las causas son múltiples; unas, inherentes al propio método, y otras, atribuibles a diversos factores ajenos al mismo. De todo ello se ha hecho ya una exposición previa (2), habiéndose llegado a la conclusión de que, no por más drástico el ensayo del autoclave es el más seguro en todos los casos, ni con todos los cementos, en cuanto a que los resultados de tal ensayo reflejen el comportamiento real, diferido o no, de los conglomerados hidráulicos en la práctica. Factores tales como las propias condiciones experimentales y operatorias del método, el "efecto silícico o sílicocalcáreo", y la activación puzolánica hidrotérmica a alta temperatura, unidos a la presencia de "componentes secundarios" activos o activables en los cementos portland "comerciales" y en los cementos especiales a base de clínker portland, pueden impedirlo.

Se trata ahora de exponer aquí otro tipo de limitación, no imputable al ensayo de autoclave, aunque sí digna de tenerse en cuenta: la falta de correlación entre el valor de la expansión, el contenido de cal libre, el contenido de magnesia, el contenido de aluminatos cristalinos, y la suma de estos tres contenidos en los cementos. Esta es una conclusión a la que se ha llegado siempre que se ha tratado de establecer tales correlaciones, y que se ha podido confirmar una vez más en este trabajo, a base de una amplia estadística de datos recientes.

\subsection{Parte experimental}

\subsection{Materiales}

Los cementos objeto de este estudio han sido cerca de 600, que a lo largo de los últimos ocho años han pasado por la Sección de Análisis y Ensayos, del Departamento de Quí- 
mica del I.E.T.c.c.* En su gran mayoría se trata de cementos portland (o portland "comerciales").

\subsection{Método de análisis de cal libre}

Se ha seguido para la determinación de la cal libre de los cementos el método de las normas españolas (3), trasunto del de las normas ASTM (4). Este método, llamado de la glicerina-etanol, pone de manifiesto conjuntamente la cal libre que se encuentra como óxido $\mathrm{CaO}$ y como hidróxido $\mathrm{Ca}(\mathrm{OH})_{2}$.

\subsection{Método de determinación de la expansión}

Se ha seguido el correspondiente de las normas ASTM (1).

\subsection{Resultados}

En el Cuadro 1 se representan, a manera de gráfico, en horizontal ("abscisas") valores de cal libre en tanto por ciento, de décima en décima, desde $0 \%$ hasta $4,5 \%$; y en vertical ("ordenadas") los intervalos de clase de la expansión en autoclave, en tanto por ciento, de $0,05 \%$ en $0,05 \%$, desde valores negativos (retracción) de $-0,10 \%$ a $-0,05 \%$ hasta $1,35 \%$ a $1,40 \%$. Verticalmente se totaiizan los cementos correspondientes a cada tanto por ciento de cal libre, y horizontalmente los correspondientes a cada intervalo de clase de la expansión. También se totalizan en cada caso, por una parte, los cementos correspondientes a contenidos de cal inferiores a $1 \%$, los comprendidos entre $1 \%$ y $2 \%$, entre $2 \%$ y $3 \%$, entre $3 \%$ y $4 \%$ y entre $4 \%$ y $4,5 \%$; y, por otra parte, los cementos retractivos con expansión negativa comprendida entre $-0,10 \%$ y $0,00 \%$, y los expansivos con expansión comprendida entre $0,00 \%$ y $0,50 \%$, entre $0,50 \%$ y $1,00 \%$, entre $1,00 \%$ y $1,40 \%$, y superior a $1,40 \%$. Estas totalizaciones se aprecian globalmente en el Cuadro 2.

El Cuadro 1, equivalente a un gráfico, muestra una nube de puntos muy extendida, pero que presenta una concentración de ellos tanto mayor cuanto menor es el contenido de cal libre y al mismo tiempo cuanto menor es la expansión en autoclave. Estos mismos resultados los ofrecen respectivamente y por separado los diagramas de frecuencia de las figuras 1 y 2 . En ellos pueden apreciarse frecuencias elevadas para contenidos de cal del orden de $0,5 \%$ y $1,5 \%$ (máxima alrededor de $0,8 \%$ ) y para expansiones del orden de $0 \%$ a $0,3 \%$ (máxima alrededor de $0,1 \%$ ).

Las figuras 3 y 4 presentan los valores de las frecuencias, por intervalos de clase, para la cal libre y para la expansión, respectivamente, correspondientes al Cuadro 2.

\subsection{Interpretación $y$ discusión}

El cuadro y gráficos anteriores muestran un hecho claro: estadísticamente, y dentro de la población considerada, son mucho más frecuentes los casos en los que a un contenido pequeño de cal libre corresponde una expansión en autoclave también exígua. Esto está dentro de la lógica y de lo que razonablemente cabe esperar, puesto que debe haber una proporcionalidad directa entre el contenido de un agente expansivo en el cemento y la expansión producida por el mismo, con independencia de la forma de la posible relación matemática que ligue a ambas variables.

Sólo aquellos cementos de los que se tenía simultáneamente el valor de la cal libre y el de la. expansión en autoclave. 
No obstante, cabría esperar, en principio, una relación sencilla y mejor definida, incluso lineal, entre el contenido de cal libre y la expansión. La dispersión de los Cuadros 1 y 2 indica lo contrario, pues no faltan valores de cal libre inferiores a $1 \%$ a los que corresponden expansiones superiores a $1,4 \%$, ni valores de cal libre superiores a $4 \%$ a los que corresponden expansiones inferiores a $0,5 \%$. Por el contrario, y paradójicamente, faltan valores de cal libre superiores a $4 \%$ a los que correspondan expansiones superiores a $1,4 \%$.

Cuando se trata de analizar las posibles causas de estos hechos aislados y relativamente poco numerosos, pero evidentemente anómalos en apariencia, se encuentran varios motivos de muy diversa naturaleza. Unos se refieren al método de determinación de la expansión, y otros a los métodos analíticos y de cálculo para la determinación de cal libre, de magnesia total y cristalizada, y de aluminatos totales y cristalinos.

En cuanto al método de determinación de la expansión, dado su carácter superdrástico, puede poner de relieve la expansión total debida en conjunto a los tres agentes expansivos principales citados en la introducción. Se dice "puede poner" y no "pone", porque hay casos en que, como se indica en el apartado 2, el "efecto silícico o sílico-calcáreo" y la activación puzolánica dan lugar a un enmascaramiento de la expansión precisamente debida a la cal libre. Así, pues, en estos casos, aun tratándose de cementos con cal libre, la totalidad o una parte mayor o menor de la expansión debida a ella puede quedar oculta (2). El ensayo de autoclave, sólo pondrá de manifiesto en tales casos la expansión debida a la periclasa y/o a los aluminatos cristalinos.

Por lo que respecta a los métodos para la determinación de la cal libre, la generalidad de ellos, y en particular el método de la glicerina-etanol (4), dan por resultado la suma de $\mathrm{CaO}$ y $\mathrm{Ca}(\mathrm{OH})_{2}$ presentes en el cemento, siendo así que de ambos compuestos sólo el primero - cal- es capaz de dar expansión al transformarse en el segundo - portlandita-. Solo una deter minación no química, sino basada en métodos de naturaleza física, tales como el microscópico (5), podría proporcionar el valor verdadero del contenido de $\mathrm{CaO}$, como tal, tanto más interesante cuanto que éste y no el $\mathrm{Ca}(\mathrm{OH})_{2}$ ni la suma $\mathrm{CaO}+$ $+\mathrm{Ca}(\mathrm{OH})_{2}$, es el responsable de la expansión debida a la cal libre. Otro método analítico que permitiría distinguir cuantitativamente entre $\mathrm{CaO}$ y $\mathrm{Ca}(\mathrm{OH})_{2}$ en presencia, sería el térmico diferencial.

En cuanto al óxido cálcico libre, $\mathrm{CaO}$, su acción expansiva correspondiente a un contenido dado de él en el cemento, depende, no sólo de dicho contenido, sino de la naturaleza y estado del $\mathrm{CaO}$ en el clínker, esto es, de que se trate de cal libre primaria o secundaria, así como del tamaño de grano cristalino de dicha cal, de su inclusión o no en los cristales de otros componentes del clínker, y de la mayor o menor temperatura a la que dicha cal se haya formado o se haya visto sometida en el horno de cemento. Los efectos de la expansión pueden depender mucho, en la práctica, de tales factores, los cuales pueden influir también cualitativa e incluso cuantitativamente en los resultados de la expansión en autoclave.

En lo relativo a la determinación de la magnesia, el método químico ordinario pone de manifiesto el contenido total de $\mathrm{MgO}$ del cemento, sin discriminar si se trata de magnesia vítrea o amorfa, o de periclasa cristalina, o de la mezcla de ambas, siendo así que sólo la periclasa es activa a efectos de expansión al transformarse en brucita por hidratación. Como en el caso de la cal libre, sólo un método de carácter físico, como por ejem- 
plo el microscópico, sería capaz de dar el contenido de periclasa, el cual guardaría relación con la expansión en autoclave.

Lo mismo sucede con los aluminatos del cemento, y más concretamente con el $\mathrm{C}_{3} \mathrm{~A}$. Este componente, o bien se evalúa a base del análisis químico, aplicando el cálculo de la composición potencial, o bien se determina por vía microscópica, mediante recuento. En el primer caso resulta un aluminato tricálcico total "teórico" (y del mismo modo se puede obtener por cálculo el ferrito-aluminato $\mathrm{C}_{4} \mathrm{AF}$ o la disolución sólida $\mathrm{C}_{6} \mathrm{AF}_{2}$ ), que engloba tanto el aluminato cristalino como el amorfo o vítreo. Hay que tener en cuenta que, puesto que el cálculo potencial se basa en la cristalización en condiciones de equilibrio térmico de los componentes del clínker, el contenido real de aluminatos (y de ferrito-aluminatos) cristalizados se aproximará tanto más al valor potencial calculado, cuanto más lentamente se haya enfriado el clínker, cosa que no suele ser frecuente en la fabricación de cemento, sino todo lo contrario. De aquí que el $\mathrm{C}_{3} \mathrm{~A}$ y/o el $\mathrm{C}_{4} \mathrm{AF}$ (o el $\mathrm{C}_{6} \mathrm{AF}_{2}$ ) potenciales puedan no estar, en general, en relación con la expansión en autoclave.

La determinación microscópica de aluminatos de forma global es posible y fácil, y hasta permite, a veces, distinguir la proporción de compuestos cristalinos, únicos responsables principales de la expansión debida a los aluminatos. En este sentido, el método microscópico es el más idóneo.

En cuanto al método difractométrico de rayos $\mathrm{X}$, específico para detectar sustancias cristalizadas, su empleo en la determinación de $\mathrm{MgO}$ y de $\mathrm{C}_{3} \mathrm{~A}$ es de eficacia dudosa, dado su carácter semicuantitativo y la pequeña proporción en que suele encontrarse la magnesia en el cemento. Contribuye a dificultar su aplicación, el estado a veces microcristalino o seudoamorfo en que se presentan en parte ciertos componentes, lo cual provoca respuestas a la radiación, por parte de éstos, distintas de cuando se encuentran en estado francamente cristalino.

Todo lo que antecede explica la lógica falta de correlación entre las expansiones (totales) en autoclave de los cementos y sus contenidos de cada uno de los compuestos expansivos principales (por separado), así como incluso entre las expansiones y las sumas de dichos contenidos. En el presente trabajo queda probado esto, por lo que se refiere a la cal libre; en otros sucesivos se tratará de probar lo mismo respecto de la magnesia, de los aluminatos, y de la suma de ambos más la cal libre.

\section{CONCLUSIONES}

No se puede pretender, en general, encontrar una buena correlación entre los valores de expansión en autoclave de los cementos y sus respectivos contenidos de cal libre:

a) porque la expansión total que se determina con el ensayo de autoclave no depende sólo de la cal libre, sino también de la periclasa y de los aluminatos cristalizados;

b) porque la cal libre que se relaciona o debe relacionarse con la expansión no es la cal libre total -óxido $\mathrm{CaO}$ más hidróxido $\mathrm{Ca}(\mathrm{OH})_{2}$ - que proporcionan los métodos analíticos químicos (y concretamente el de la glicerina-etanol), sino la cal libre en forma de óxido - cal viva, no hidratada-;

c) porque el efecto expansivo de esta cal libre $\mathrm{CaO}$ no depende sólo de su cuantía, sino de la forma en que se encuentra en el cemento, como consecuencia de su génesis y estado en el clínker. 


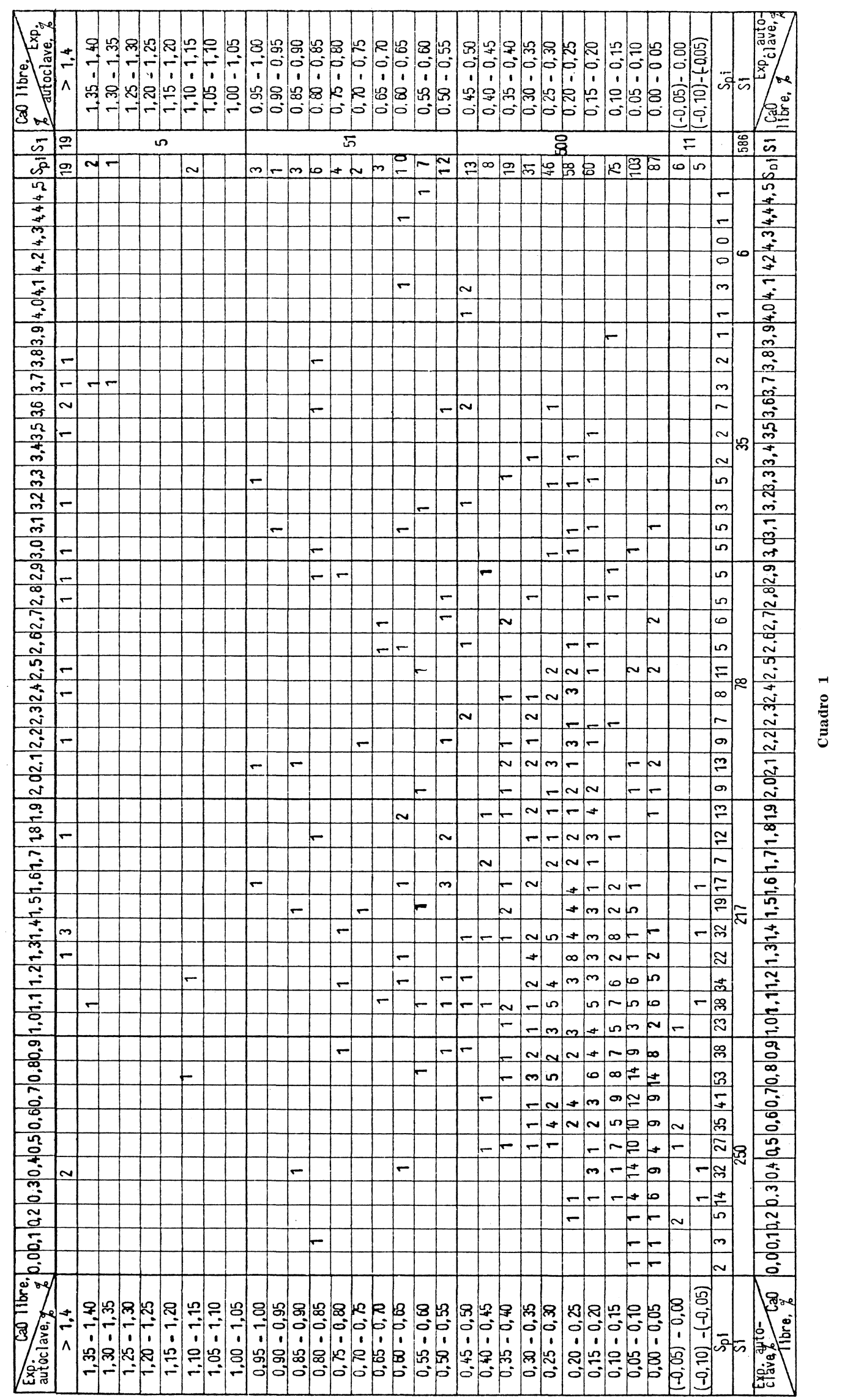




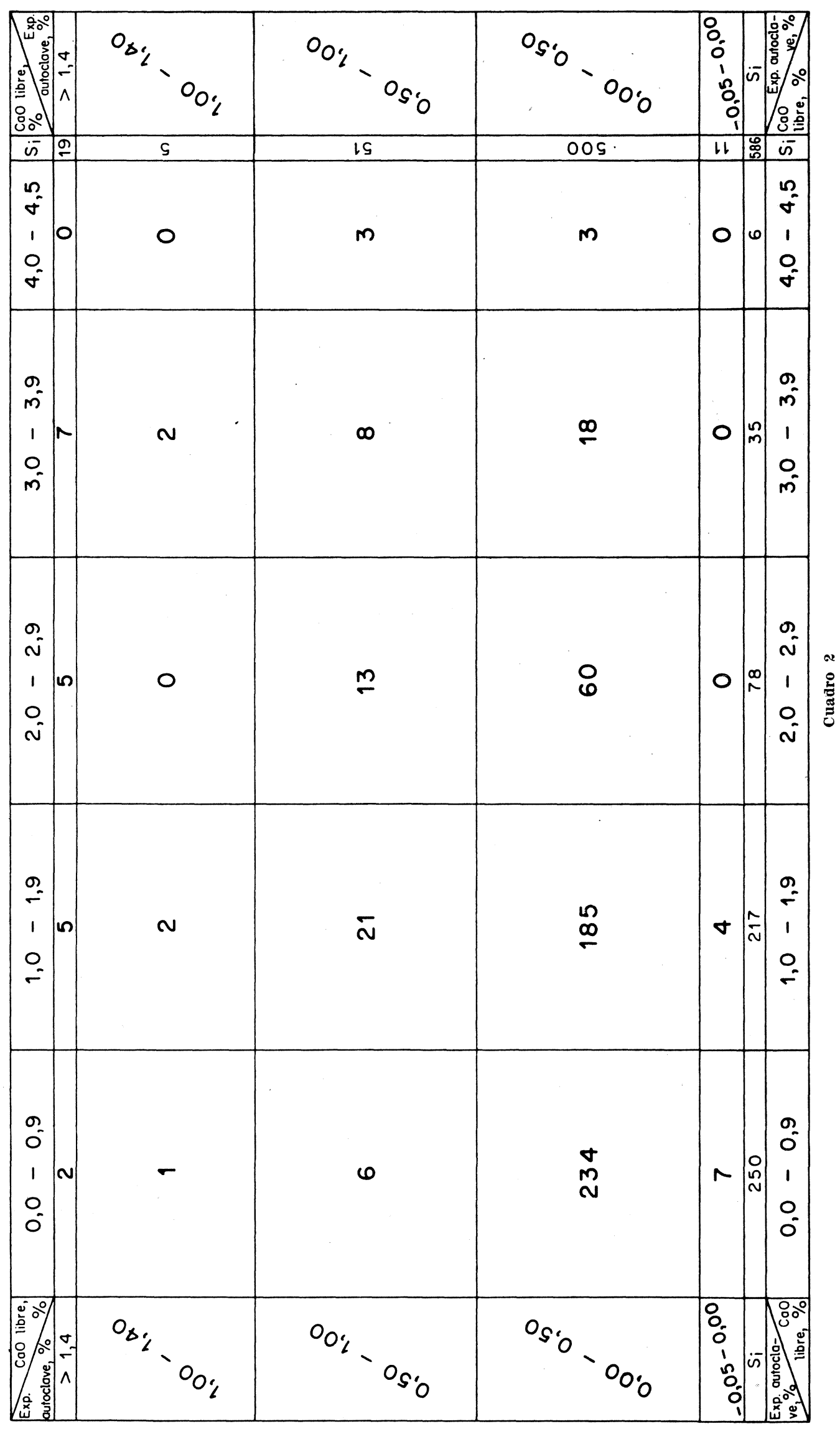



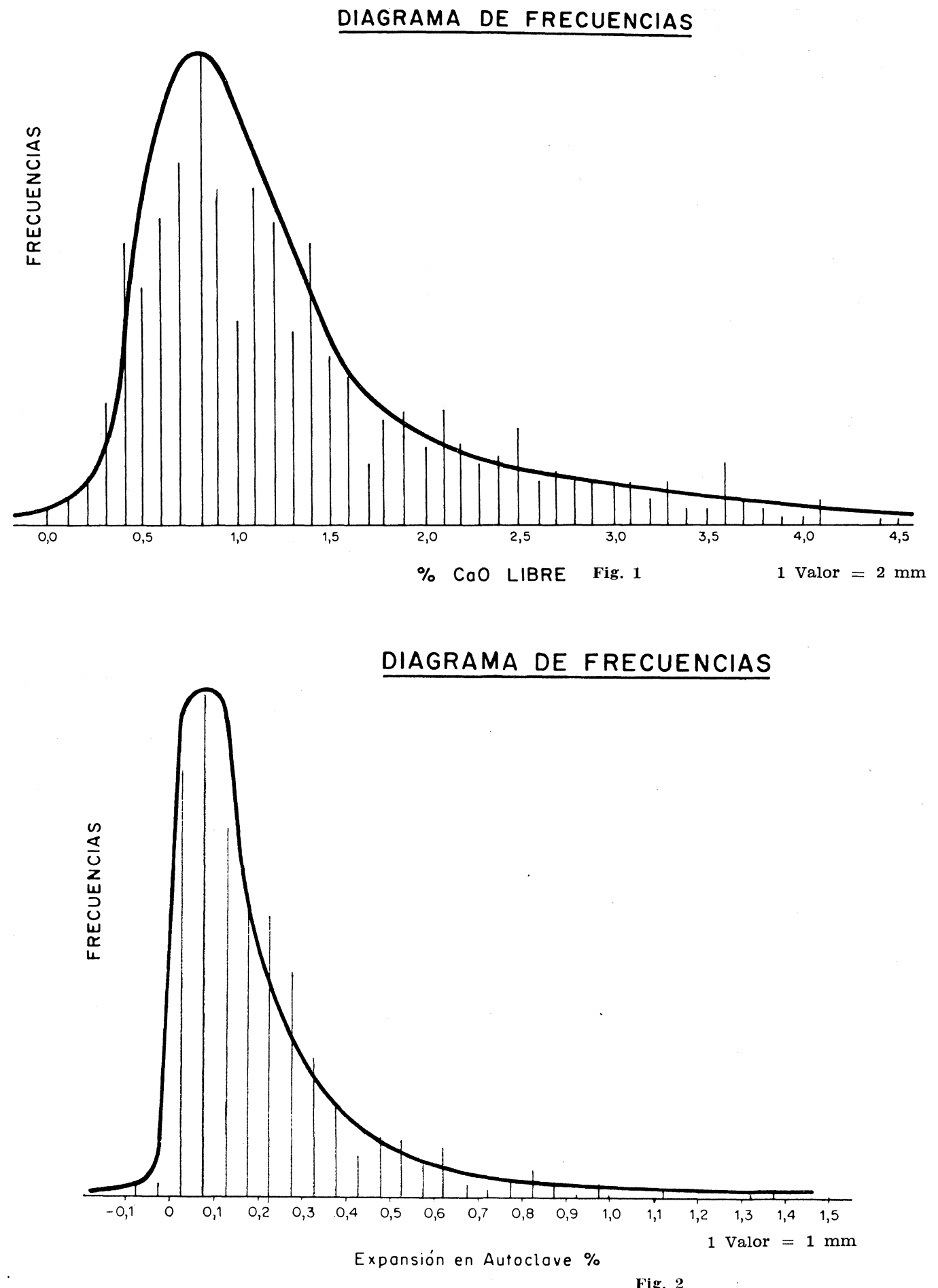

A partir de estos resultados y de su interpretación y discusión, cabe esperar que suceda otro tanto con respecto a la magnesia y a los aluminatos, así como a la suma de $\mathrm{CaO}$ libre, $\mathrm{MgO}$ y $\mathrm{C}_{3} \mathrm{~A}$ (y eventualmente $\mathrm{C}_{4} \mathrm{AF}$ o $\mathrm{C}_{6} \mathrm{AF}_{2}$ ), extremos que serán objeto de consideración en trabajos sucesivos.

\section{R E F E R E N C I A S}

(1) Normas Norteamericanas (U.S. A.).: ASTM Standard C 150-67 y ASTM Standard Method of Test C 151-68 for Autoclave Expansion of Portland Cement. 


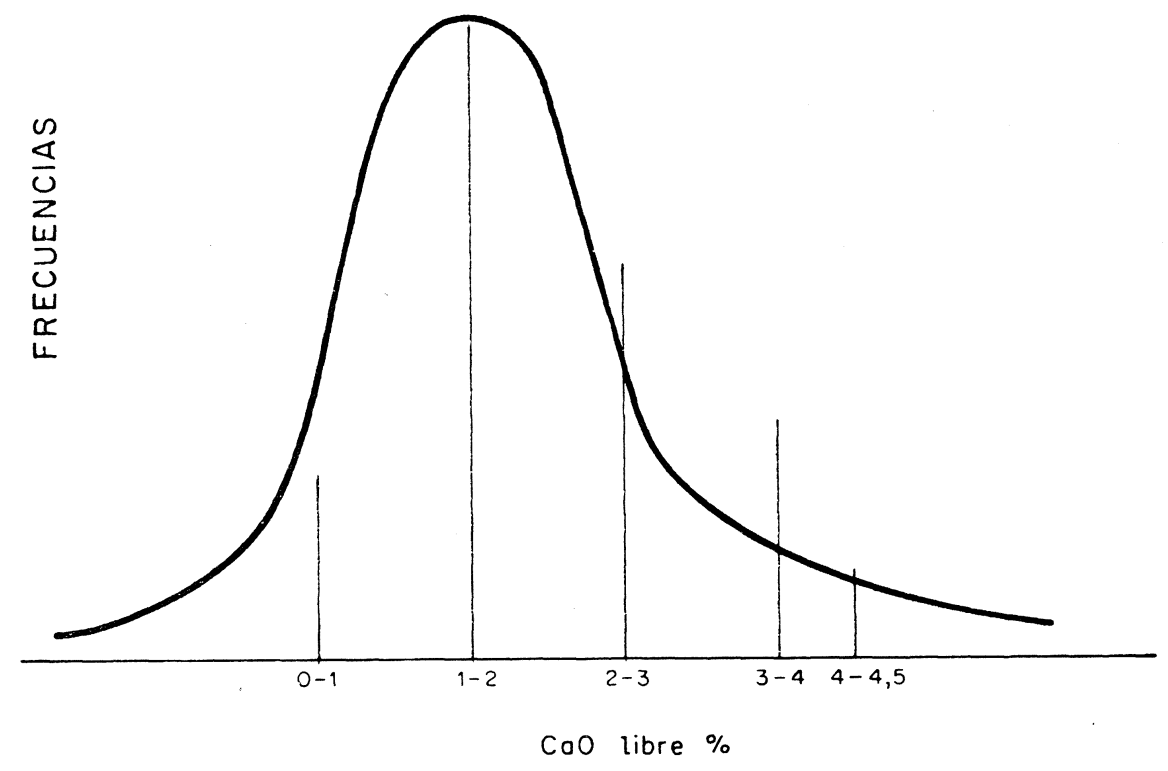

INTERVALOS

Fig. 3

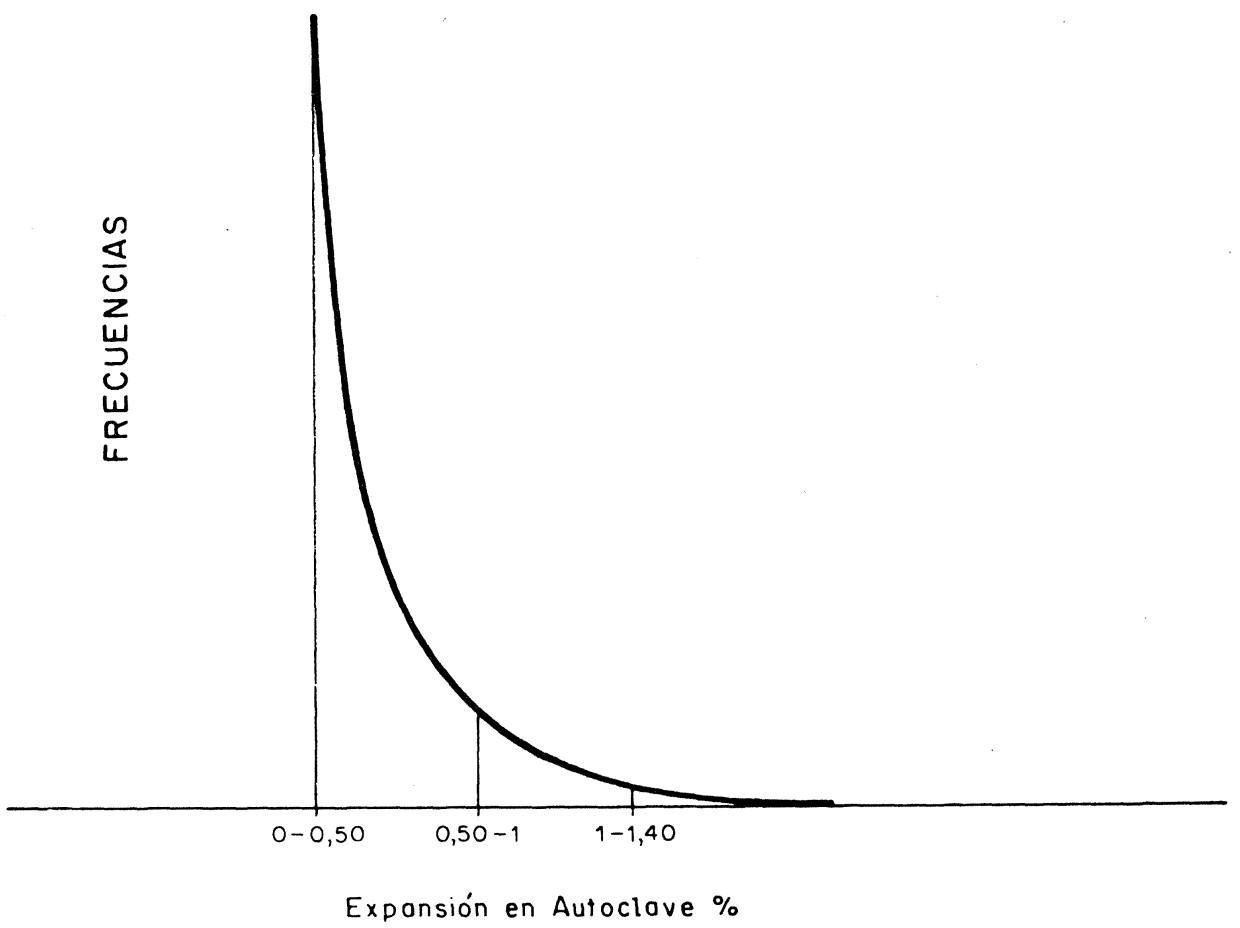

INTERVALOS Fig. 4

(2) Calleja, J.: "Sobre la expansión de los cementos y los métodos para su determinación. (Limitaciones lógicas del campo de aplicación y de la validez de los resultados de ensayos de expansión de cementos en autoclave)". Materiales de Construcción (Ultimos Avances) I.E.T.c.c., núm. 141, ene.-feb.mar. 1971.

(3) P.C.C.H.-64.: Normas Españolas para Cemento (I.E.T.c.c.) 1964, Madrid.

(4) Normas Norteamericanas (U.S. A.).: ASTM Standard Method of Test C 114-69 for Chemical Analysis of Hydraulic Cement.

(5) Coronas, J. M.: "Técnicas aplicadas a la investigación del cemento". Curso de Especialización en Cemento (I.E.T.c.c.) Madrid, 1950. 